Check for updates

Cite this: RSC Adv., 2021, 11, 1411

Received 28th September 2020 Accepted 18th December 2020

DOI: $10.1039 / \mathrm{d} 0 \mathrm{ra0} 8287 \mathrm{~b}$

rsc.li/rsc-advances

\section{A novel green synthesis of silver nanoparticles by the residues of Chinese herbal medicine and their biological activities $\uparrow$}

\author{
Simin Wei, (D) a Yinghui Wang, (D) ${ }^{* b}$ Zhishu Tang, ${ }^{\star a}$ Hongbo Xu, ${ }^{a}$ Zhe Wang, ${ }^{a}$ \\ Tian Yang ${ }^{a}$ and Taiyan Zou ${ }^{a}$
}

Green synthesis of silver nanoparticles (AgNPs) by using the extracts of Chinese herbal medicines (CHMs) has attracted tremendous attention due to the potential synergistic effect between metal nanoparticles and capping agents. However, since $\mathrm{CHMs}$ are precious and expensive, finding other cheap and ecofriendly resources for biosynthesizing AgNPs with superior medicinal activites is necessary. Herbal medicine residues (HMRs) are the by-products of traditional Chinese herbal medicine after decoction and were identified to contain approximately 30-50\% of medicinally active ingredients, which may be advantageous for green synthesis of medicinal AgNPs. Inspired by this, we present herein the preparation of AgNPs by reusing Bazheng Mixture residues and evaluate both biosynthesis parameters and bioactivities, where Bazheng Mixture is a famous Chinese patent medicine for relieving inflammation and pain, and allaying fever. The UV-visible spectrum and DLS analysis showed that the as-prepared AgNPS were sensitive to $\mathrm{pH}$, material proportion and incubation time, but the yield was impervious to material proportion. TEM, HRTEM, SAED and DLS characterization found that AgNPs ( $\mathrm{pH}$ 10.0; material proportion $1: 1 ; 6 \mathrm{~h}$ ) had a face-centered cubic ( $\mathrm{fcc}$ ) structure and spherical shape with an average size of $22.2 \pm 0.5 \mathrm{~nm}$ covered by anions, and existed in monodispersed form with long term stability. The AgNPs displayed potent toxic effects against both cancer cell lines and pathogens, and superior antioxidant activity. The $\mathrm{IC}_{50}$ for HCT116, HepG2 and HeLa cell lines were 13.07, 19.67, and $26.18 \mu \mathrm{g}$ $\mathrm{mL}^{-1}$, respectively. The MICs of AgNPs for E. coli and S. aureus were both $50.0 \mu \mathrm{g} \mathrm{mL}^{-1}$. The uptake analysis of AgNPs for both pathogens and cancer cell lines was performed to preliminarily illustrate the mechanism of toxic effects. These results confirm that HMRs could be a low-cost, nontoxic and ecofriendly resource for green synthesis of medicinal AgNPs, and also provide an alternative method for general recycling strategies of HMRs.

\section{Introduction}

Due to the wide use of silver nanoparticles (AgNPs) in the field of biomedicine, electronics, biosensors and catalysis, analysts have focused tremendous attention on the synthetic approaches like autoclaving, gamma-ray radiation, electrochemical techniques and chemical reduction. ${ }^{1-6}$ Although a high yield of AgNPs could be obtained by utilizing these traditional synthetic approaches, simultaneously they also bring in toxic reducing and capping agents or consume lots of energy, which limit

${ }^{a}$ Shaanxi Collaborative Innovation Center of Chinese Medicine Resources Industrialization, State Key Laboratory of Research \& Development of Characteristic Qin Medicine Resources/Shaanxi Innovative Drug Research Center, Shaanxi University of Chinese Medicine, 712046, China.E-mail: tzs6565@163.com ${ }^{b}$ College of Science, Chang'an University, 710064, China.E-mail: wangyinghui@iccas. ac.cn; wangyinghui@chd.edu.cn

$\dagger$ Electronic supplementary information (ESI) available. See DOI: $10.1039 /$ dora08287b a wide range of AgNPs use especially in biomedicine. To overcome these drawbacks of physiochemical methods, plant extracts, bacteria, fungi and biopolymers have been used for developing alternative simple, cost-effective, safe, and ecofriendly synthetic methods of AgNPs. ${ }^{7-13}$ Usually, the less biothreatening plant-based synthesis was adopted more frequently compared to these methods by using microorganisms and biopolymers as it could be improved easily, and did not include the step of cell culture growth. In the process of plant-based synthesis, biological molecules, including terpenoids, flavones, ketones, aldehydes, amides, carboxylic acids, carbohydrates, proteins, and vitamins etc., could act as not only the reducing but also capping agents. ${ }^{8,14}$ These capping agents are considered to prevent the nanoparticles from agglomeration and reduce the toxicity. It has been widely accepted that if these coating agents themselves show biological activities, a synergistic effect between metal nanoparticles and these capped molecules would be anticipated. To date, several plant including Coconut inflorescence sap, ${ }^{13}$ Citrus reticulata peels, ${ }^{15}$ 
grape seed, ${ }^{\mathbf{1 6}}$ Arbutus unedo, ${ }^{\mathbf{1 7}}$ Nelumbo nucifera, ${ }^{\mathbf{1 8 , 1 9}}$ Fumaria parviflora $^{\mathbf{2 0}}$ and Coffea arabica ${ }^{\mathbf{1 0}}$ have been employed for biosynthesizing AgNPs with different physicochemical and biological properties. Especially, since it is rich of bioactive ingredients, traditional Chinese herbal medicines (CHMs) like Sea buckthorn, ${ }^{21}$ Cornus officinalis, ${ }^{22}$ Iresine herbstii, ${ }^{23}$ Sesbania grandiflora $^{24}$ and Alpinia katsumadai ${ }^{25}$ have also attracted enormous attentions for biosynthesizing medicinal AgNPs.

However, CHMs have been extensively applied for health care by about $80 \%$ of populations and developed the essential part of healthcare system in China ${ }^{26,27}$ as the important roles in treating many chronic diseases, cardiovascular diseases, and in the drug development. ${ }^{28}$ In recent years, it has been considered as an alternative or complementary medical treatment worldwide. $^{26,29}$ These prosperous developments make CHMs precious and expensive. Thus, to seek other cheap and eco-friendly resources for biosynthesizing AgNPs with superior medicinal activities is necessary.

Herbal medicine residues (HMRs) are the by-products of traditional CHMs after decocting with water or ethanol, which is estimated that tens of million tons herb residues were generated in $2015 .^{30}$ Generally, it has been identified that approximately $30-50 \%$ of the medicinally active ingredients, mainly including abundant alkaloids, flavonoids, fat, amino acids, protein and polysaccharides, ${ }^{31}$ are still presented in HMWs. These remaining bioactive ingredients reveal a wide range of bioactivities such as anti-inflammatory, anti-oxidative, anti-neoplastic and antidiabetic activities. On this basis, it is possible to prepare AgNPs with superior bioactivities by using HMWs.

Bazheng Mixture is a famous Chinese patent medicine, which has the effects of relieving inflammation and pain, and allaying fever. ${ }^{32}$ Its chief ingredients are Semen Plantagini, Herba Dimathus superbus, Herba Polygoni avicularis, Talcum, Fructus Gardenia jasminoides, Radix Glycyrrhiza praeparata, Caulis Akebiae, Radix et Rhizoma rhei and Medulla Junci. A large number of residues have also been generated after producting this recipe. In this study, we were motivated to develop a novel and eco-friendly green synthesis of AgNPs by utilizing the residues of Bazheng Mixture (RBM) under ultrasonic radiation at ambient temperature (Scheme 1). The effects of biosynthesis parameters, such as $\mathrm{pH}$, material proportion and incubation
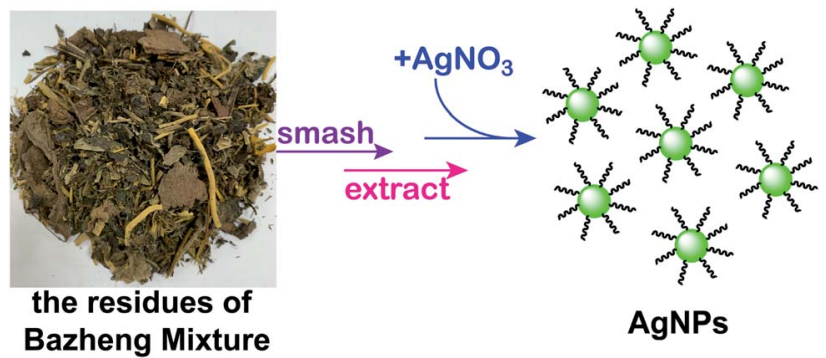

nn residual phytomolecules

$\mathrm{Ag}$

Scheme 1 Schematic of the green synthesis for AgNPs by reusing Bazheng Mixture residues as bioreductant.
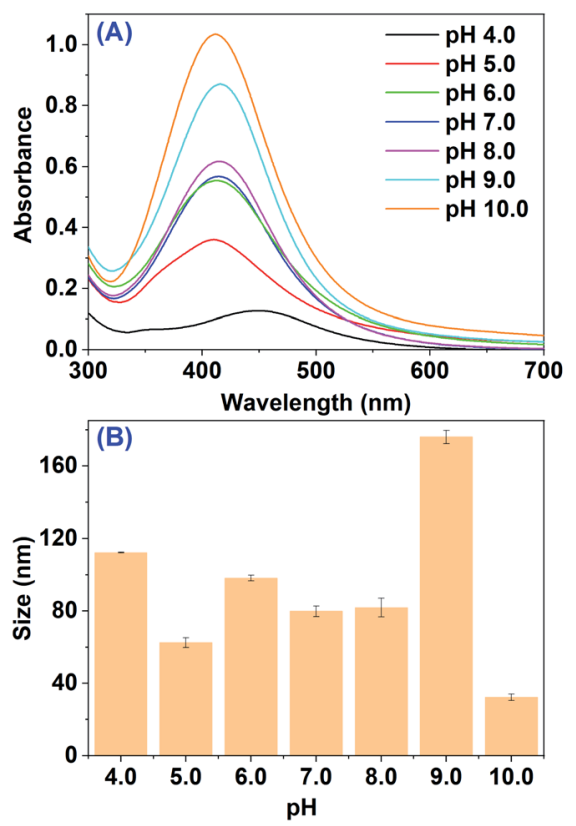

Fig. 1 (A) UV-vis spectra of mixture after the reaction between RBM extract and $\mathrm{AgNO}_{3}$ at different $\mathrm{pH}$ values; (B) average size of AgNPs obtained from (A).

time were systematically assessed by using UV-visible spectrum and dynamic light scattering (DLS). The shape, size, crystalline nature, surface properties and the stability of as-prepared AgNPs were further characterized by transmission electron microscopy (TEM), HRTEM, selected area electron diffraction (SAED), DLS and FTIR. The radical scavenging rate for DPPH, the cell viabilities for three cancer cell lines, and the minimum inhibition concentration (MIC) values for $E$. coli and $S$. aureus were calculated to disclose the bioactivities of as-prepared AgNPs. Also, to preliminarily illustrate the mechanism of cell death, the uptake analysis of AgNPs into both bacteria and cancer cells were performed by calculating the values of side scatter (SSC). These results not only reveal that the RBM could be used to biosynthesize AgNPs, but also provide a novel method for general recycling strategies of HMRs.

\section{Experimental}

\subsection{Materials}

The residues of Bazheng Mixture (RBM) were obtained from Shaanxi Institute of Traditional Chinese Medicine Pharmaceutical Factory (Xianyang, China). Silver nitrate $\left(\mathrm{AgNO}_{3}, 99.8 \%\right)$, sodium chloride, tryptone, yeast and agar powder were bought from Chron Chemicals Co., Ltd (Chengdou, China). 1,1-Diphenyl-2picrylhydrazulin (DPPH, >99.5\%) was bought from Shanghai Aladdin Bio-Chem Technology Co., Ltd. 3-(4,5-Dimethyl-2-thiazolyl)-2,5-diphenyl-2H-tetrazolium bromine (MTT) and dimethyl sulfoxide (DMSO) were bought from Solarbio Science \& Technology Co. Ltd (Beijing, China). Other reagents used in experiments were bought from Sigma Aldrich. Human colorectal cancer HCT116, human hepatoma cancer HepG2 and cervical cancer HeLa cell lines were bought from the cell repository of the Chinese 
Academy of Sciences (Shanghai, China). All solutions were prepared in ultrapure Milli-Q water. All chemicals were of analytical grade and used without further purification.

\subsection{Synthesis of silver nanoparticles}

To obtain the RBM extract, the RBM (5.0 g, powder) was added into $100.0 \mathrm{~mL}$ of deionized water accompanying with sonicate for $4 \mathrm{~h}$, which was further filtered and stored in $4{ }^{\circ} \mathrm{C}$ for the following studies. The AgNPs were prepared by mixing RBM extract and $10 \mathrm{mM} \mathrm{AgNO}$ with indicated amounts under ultrasonic radiation at ambient temperature. The AgNPs were purified by centrifuging the mixture for $30 \mathrm{~min}$ at $10000 \mathrm{rpm}$. The collected precipitate was washed with deionized water three times and then lyophilized for $12 \mathrm{~h}$. The effects of extract $\mathrm{pH}$ (4.0, 5.0, 6.0, 7.0, 8.0, 9.0 and 10.0), material proportion ( $2: 1$, $1: 1,1: 2,1: 3$ and $1: 5)$ and incubation time were evaluated as per the procedures mentioned above.

\subsection{Characterization of silver nanoparticles}

The formation and growth of AgNPs synthesized by RBM extract under ultrasonic radiation was periodically monitored by collecting UV-vis spectra in the range of $200-800 \mathrm{~nm}$. The transmission electron microscopy (TEM) measurements were performed to characterize the morphology of AgNPs. The crystalline nature of AgNPs was examined by HRTEM and selected area electron diffraction (SAED). The average sizes and surface loads of AgNPs were measured using a Malvern Zetasizer Nanoseries (ZEN 3600, Malvern, UK) and FTIR. The Zeta potential was evaluated at ambient temperature with an angle of $17^{\circ}$ and a dielectric constant of 78.5. The electrical field was operated at $15 \mathrm{~V} \mathrm{~cm}^{-1}$. Data obtained were analyzed with Zetasizer software.

\subsection{Antioxidant activity of silver nanoparticles}

The radical scavenging activity of synthesized AgNPs was evaluated on the basis of bleaching absorbance of DPPH at $517 \mathrm{~nm}$ in ethanol. Serial concentrations $\left(0.8-200.0 \mu \mathrm{g} \mathrm{mL}^{-1}\right)$ of AgNPs $(1.0 \mathrm{~mL})$ were prepared and then added to $1.0 \mathrm{~mL}$ of $\mathrm{DPPH}$ solution $\left(2.5 \mathrm{mg} \mathrm{mL}^{-1}\right)$. After the reaction mixtures were vigorously shaken and incubated for 30 minutes in the dark at room temperature, the absorbance of mixture at $517 \mathrm{~nm}$ was measured, where a negative control group (without AgNPs) was also prepared. The radical scavenging rate was calculated according to the given formula:

$$
\text { Scavenging rate }(\%)=\left[1-\left(A_{1}-A_{2}\right) / A_{0}\right] \times 100 \%
$$

where $A_{0}$ is the absorbance of DPPH and ultrapure water at $517 \mathrm{~nm}, A_{1}$ is the absorbance of DPPH and AgNPs at $517 \mathrm{~nm}$, and $A_{2}$ is the absorbance of ethanol and AgNPs at $517 \mathrm{~nm}$.

\subsection{Antibacterial activity of silver nanoparticles}

To test the toxic effect of AgNPs synthesized by RBM extract on both Gram positive ( $S$. aureus) and Gram negative (E. coli) bacteria, the growth inhibition studies were conducted in Luria-Bertani (LB) broth media. The experiments were performed as previously reported methods with some modifications. ${ }^{33}$ For growth inhibition studies, 96-well plates, each containing $50 \mu \mathrm{L} \mathrm{LB}$ broth media and desired amount of AgNPs, were inoculated with $50 \mu \mathrm{L}$ of the freshly prepared bacterial suspension in order to maintain initial bacterial concentration in the same range in the well. The 96-well plates were then incubated in a rotary shaker at $160 \mathrm{rpm}$ at $37^{\circ} \mathrm{C}$. The growth of these two pathogens was monitored every hour for $24 \mathrm{~h}$ by measuring the absorbance at $600 \mathrm{~nm}$. A control experiment containing only media and bacteria devoid of AgNPs was also included.

\subsection{Anticancer activities of silver nanoparticles}

Human colorectal cancer HCT116, human hepatoma cancer HepG2 and human breast cancer Hela cell lines were cultured at $37{ }^{\circ} \mathrm{C}$ in a humidified atmosphere containing $5 \% \mathrm{CO}_{2}$ and grown continuously in DMEM medium supplemented with $10 \%$ heat in activated fetal bovine serum (FBS). Cells were plated out in the bottom of 96-well plates at a density of $1 \times 10^{4}$ cells per well and allowed to attach for $24 \mathrm{~h}$. After sucking up the supernatant, the desired amounts of AgNPs $\left(0.8-100.0 \mu \mathrm{g} \mathrm{mL}^{-1}\right)$ were added into 96-well plates. The cells were then incubated at $37{ }^{\circ} \mathrm{C}$ in an atmosphere of $5 \% \mathrm{CO}_{2}$ for $24 \mathrm{~h}$ followed by an MTT assay. $20 \mathrm{~mL}$ Thiazolyl Blue Tetrazolium Bromide (MTT) $(5.0 \mu \mathrm{g}$ $\mathrm{mL}^{-1}$, Solarbio) and $150 \mathrm{~mL}$ DMEM media were freshly added to each well. After incubation for $4 \mathrm{~h}$, the media was gently removed. The formed formazan crystals were dissolved in $150 \mathrm{~mL}$ DMSO, subsequently the absorbance was measured with a plate reader (PerkinElmer) at $490 \mathrm{~nm}$. The cell viability was calculated according to the given formula:

$$
\text { Cell viability }(\%)=\left(A-A_{\mathrm{b}}\right) /\left(A_{0}-A_{\mathrm{b}}\right) \times 100 \%
$$

where $A$ is the absorbance of sample, $A_{\mathrm{b}}$ is the bleaching absorbance without sample, and $A_{0}$ is the absorbance value of control.

\subsection{Internalization of silver nanoparticles}

According to the method of Suzuki et al. ${ }^{34}$ we performed flow cytometric analysis for the assessment of AgNPs internalization in both bacteria and cancer cell lines utilizing light scattering principles. In brief, the bacteria and cancer cell lines were seeded in 6-well culture plates and exposed to 5.0 and $10 \mu \mathrm{g}$ $\mathrm{mL}^{-1}$ of AgNPs for $12 \mathrm{~h}$. After indicated incubation period, bacteria and cancer cells were harvested and resuspended. Analysis was made by using flow cytometer supplied with $488 \mathrm{~nm}$ excitation lasers. The light scattering signals were collected and detected with corresponding detection channels. Data output was analyzed utilizing the FlowJo 7.6.1 software.

\section{Results and discussion}

\subsection{The effects of biosynthesis parameters}

AgNPs were initially prepared by reducing $\mathrm{Ag}^{+}$in $5.0 \mathrm{~mL}$ of $10 \mathrm{mM} \mathrm{AgNO}_{3}$ solution with $5.0 \mathrm{~mL}$ primary extract of $\mathrm{RBM}(\mathrm{pH}$ 4.0) under ultrasonic radiation at ambient temperature. A slight 
change of the mixed solution in color from pale yellow to brown after $2 \mathrm{~h}$ incubation was noticed visually, indicating that AgNPs had been formed. Since the surface plasmon resonance (SPR) band of AgNPs are in 400-500 nm, ${ }^{25,35}$ the formation of AgNPs were further confirmed by UV-vis spectroscopy measurement, where the absorbance of $\mathrm{RBM}$ extract and $\mathrm{AgNO}_{3}$ were negligible. Fig. 1A displays the UV-vis spectroscopy of the mixture after $2 \mathrm{~h}$ incubation. The spectrum clearly displays a weak absorption band peaking at $445 \mathrm{~nm}$, which essentially resembles previous studies about the SPR peak of AgNPs. This is consistent with the observation in color by naked eyes. These results suggest that the primary extract of RBM could reduce $\mathrm{Ag}^{+}$ to AgNPs after $2 \mathrm{~h}$ incubation. It should be noticed that the spectrum also had some difference, where the resonant absorption of photons by as-prepared AgNPs was at low energy indicating a big size of AgNPs. To confirm that, the average size of biosynthesized AgNPs (112.3 $\pm 0.2 \mathrm{~nm})$ was obtained by using dynamic light scattering (DLS) method as shown in Fig. 1B and Table S1. $\dagger$ The result of DLS is in line with the observation from UV spectroscopy.

According to previous studies, ${ }^{35-37}$ the size, morphology, stability, and physicochemical properties of AgNPs, which play pivotal roles in controlling physical, chemical, optical, and electronic properties of these nanoscopic materials, would be strongly influenced by biosynthesis parameters like $\mathrm{pH}$ of extract, the material proportion and reaction time. Thus, in order to get more products with small size for further biological study, the biosynthesis parameters were evaluated systematically. We firstly tested the effect of RBM extract $\mathrm{pH}$. As shown in Fig. 1A, with pH increasing to 10.0, the SPR peak of AgNPs at $445 \mathrm{~nm}$ sharply increases accompanying with band shift between 411 to $445 \mathrm{~nm}$. The increased intensity of SPR band means the more AgNPs generated by RBM extract under ultrasonic radiation. The spectral evolution may originate from the greater ionization of biomolecules in RBM extract, which could generate strong complexing ligands for the silver ions and eventually accelerate the formation of AgNPs. The SPR band shift between $445 \mathrm{~nm}$ to $411 \mathrm{~nm}$ indicates that different size particles were formed at different $\mathrm{pH}$ value. DLS measurements further confirmed the UV-vis spectral observation. The average sizes of biosynthesized AgNPs with increased $\mathrm{pH}$ from 4.0 to 10.0 were $112.3 \pm 0.2,62.5 \pm 2.7,98.2 \pm 1.6,79.83 \pm 2.9,81.9 \pm$ $5.2,176.0 \pm 3.7$ and $32.3 \pm 1.8 \mathrm{~nm}$, respectively (Fig. 1B). The smallest sizes of nanoparticles were obtained at $\mathrm{pH}$ 10.0. This result is basically same as Sathishkumar ${ }^{33}$ and Heydari's ${ }^{38}$ viewpoint that at alkaline $\mathrm{pH}$, the synthesized AgNPs have a smaller size as compared with the nanoparticles biosynthesized at acidic $\mathrm{pH}$.

Then, the effect of material proportion between RBM extract (pH 10.0) and $\mathrm{AgNO}_{3}$ was assessed. The UV-vis spectra of mixed solution at different ratio after $2 \mathrm{~h}$ incubation at ambient temperature were obtained as shown in Fig. 2A, where the volume of $\mathrm{AgNO}_{3}(5.0 \mathrm{~mL})$ and mixture $(10.0 \mathrm{~mL})$ kept in constant. When the ratio of $\mathrm{RBM}$ extract to $\mathrm{AgNO}_{3}$ is $1: 5$, a strong band with maximum absorbance at $408 \mathrm{~nm}$ was observed. Interestingly, with the increase in material proportion of reaction mixture to $2: 1$, the peak intensity does not have any
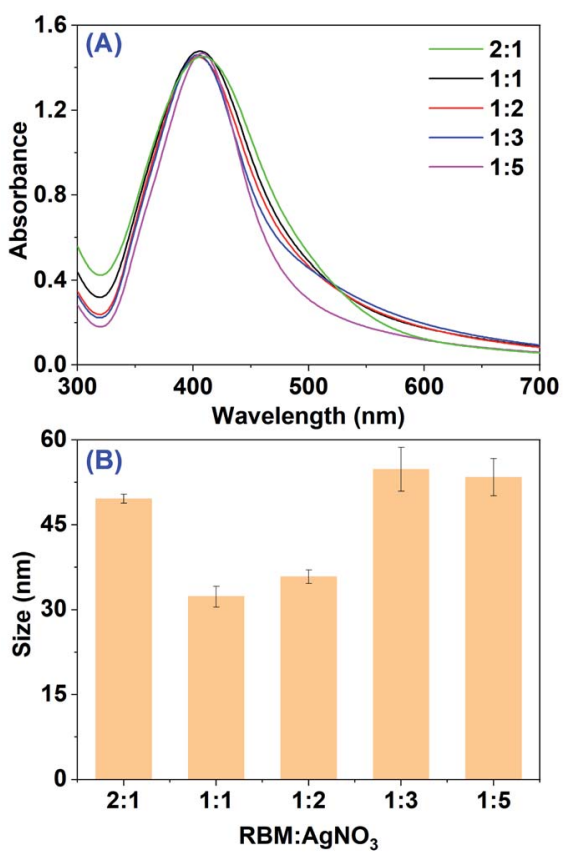

Fig. 2 (A) UV-vis spectra of mixtures after the reaction between RBM extract and $\mathrm{AgNO}_{3}$ at different ratios; (B) average size of AgNPs obtained from (A).

obvious increase. The unchanged peak intensity indicates the constant production yield of AgNPs. This may result from the ullage of $\mathrm{Ag}^{+}$in the extract after $2 \mathrm{~h}$ reaction, which was proved by following measurement of UV-vis spectra at different incubation time. Meanwhile, on increasing dose of RBM extract, a band shift between 403 to $410 \mathrm{~nm}$ was observed in the absorption spectra, meaning the different size of AgNPs. The measured average sizes by DLS at different dose of RBM extract were minimum at the proportion $1: 1(32.3 \pm 1.8 \mathrm{~nm})$ and maximum at the proportion $1: 3(54.8 \pm 3.9 \mathrm{~nm})$.

Lastly, the effect of incubation time on both biosynthesis and the size of AgNPs was tested. The absorption spectra of the mixture containing $5.0 \mathrm{~mL} \mathrm{RBM}$ extract $(\mathrm{pH} 10.0)$ and $5.0 \mathrm{~mL}$ $\mathrm{AgNO}_{3}$ recorded at different incubation times are shown in Fig. 3A. After $1 \mathrm{~h}$ incubation, a strong band at $412 \mathrm{~nm}$ corresponding to characteristic SPR band of AgNPs was clearly observed, indicating the efficient generation of AgNPs. And then, the absorption intensity increased steadily as a function of incubation time and reached a maximum after $5 \mathrm{~h}$, where no obvious band shift was displayed. The spectra evolution with increased incubation time suggests an enhancement of the biosynthesized AgNPs and the almost completed reduction reaction within $5 \mathrm{~h}$. The negligible band shift after different incubation times means the almost similar size of as-prepared AgNPs. The DLS measurements showed that the average size distribution was in the range of $22.2 \pm 0.5$ to $34.2 \pm 0.9 \mathrm{~nm}$ (Fig. $3 \mathrm{~B}$ and Table S1 $\dagger$ ) basically consistent with the observation from UV-vis spectra.

\subsection{Characterization of biosynthesized AgNPs}

A serial of experiments like TEM, HRTEM, selected area electron diffraction (SAED), DLS and FTIR for the characterizations of 

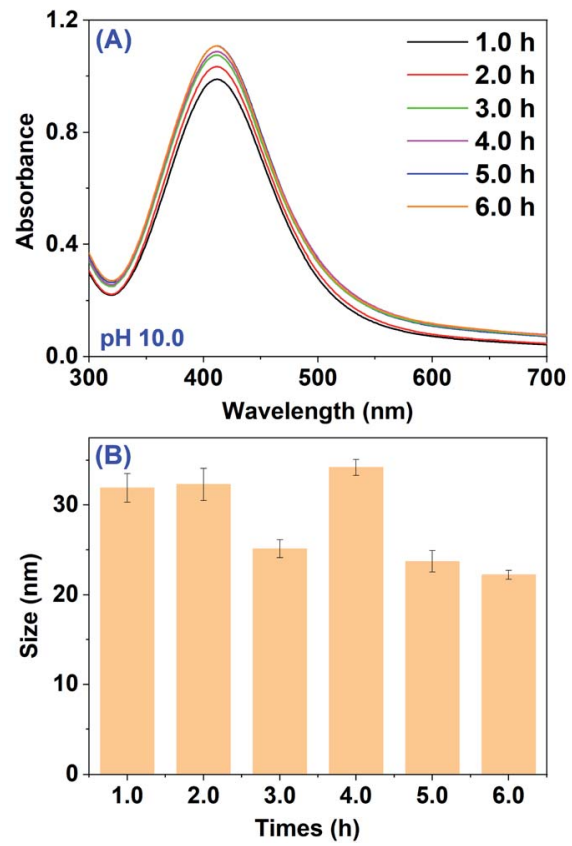

Fig. 3 (A) UV-vis spectra of mixture after the reaction between RBM extract and $\mathrm{AgNO}_{3}$ at different times; (B) average size of AgNPs obtained from (A).

biosynthesized AgNPs (pH 10.0; material proportion $1: 1 ; 6 \mathrm{~h}$ ) were carried out to confirm the proper synthesis, crystalline nature, size, shape and surface properties. Firstly, the particle size and surface morphology of as-prepared AgNPs were evaluated by using DLS and TEM. As shown in Fig. 4A and B, the biosynthesized AgNPs were mainly spherical in shape and almost all of the particles had well dispersion and scattering in nature. The diameter of AgNPs was roughly in the range of 10-
(A)

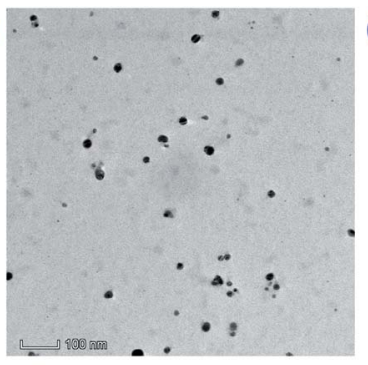

(C)

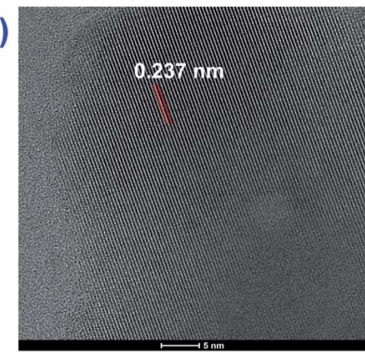

(B)

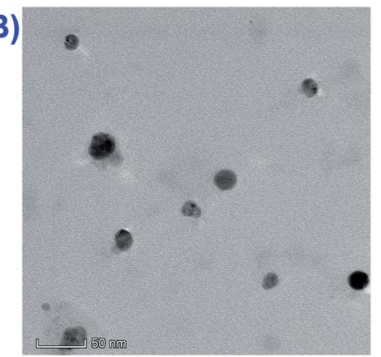

(D)

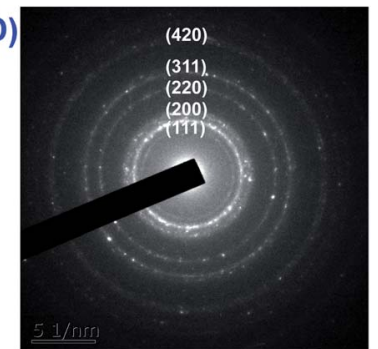

Fig. 4 (A) and (B) TEM image at different scale bar; (C) HRTEM image; (D) SAED image.
$20 \mathrm{~nm}$, which was basically in accordance with DLS's results $(22.2 \pm 0.5 \mathrm{~nm})$.

The crystalline nature of as-prepared AgNPs was then proved using the HRTEM spectrum and the SAED pattern as shown in Fig. 4C and D. The five bright circular rings with $d$ value of $2.332,2.039,1.439,1.239$ and $0.914 \AA$ observed in the SAED pattern (Fig. 4D) were the characteristic reflections of face centered cubic (fcc) crystalline silver corresponding to (111), (200), (220), (311) and (420) facets. This indicates the formation of fcc crystalline AgNPs. The crystalline nature of the biosynthesized AgNPs was further confirmed by measuring the lattice fringe, which was $2.372 \AA$ assigned to (111) facets (Fig. 4C).

Lastly, the surface state and the long-term stability of the synthesized AgNPs were predicted by measuring the zeta potential. According to previous studies, ${ }^{38,39}$ if the zeta potential of nanoparticles is outside of the range -25.0 to $+25.0 \mathrm{mV}$, they would have superior stability. In parallel, the nanoparticles with zeta potential between the interval would have interparticle attractions and eventually lead to the generation of aggregation. As shown in Fig. S1, $\dagger$ the zeta potential of biosynthesized AgNPs by RBM extract was $-25.5 \mathrm{mV}$ approximating to boundary of $-25.0 \mathrm{mV}$, indicating a high degree of stability. Practically, the average size of the biosynthesized AgNPs $(23.1 \pm 0.6 \mathrm{~nm})$ did not have any apparent changes after 2 months (Fig. S2†). The negative zeta potential also indicated the electronegative capping agents in the surface of AgNPs, which may be alkaloids, flavonoids, fat, polysaccharides, protein and amino acids contained in RBM extract. The identity of potential reducing and capping agents responsible for biosynthesized AgNPs was confirmed by further FTIR study (Fig. S3†).

\subsection{Antioxidant activities of biosynthesized AgNPs}

DPPH method was performed to accurately reflect the antioxidant properties of as-prepared AgNPs at different concentration. DPPH is a stable free radical and could accept hydrogen and electrons from the donors accompanying with a color change from purple to yellow. The dose-response bar chart of DPPH scavenging activity for AgNPs is shown in Fig. 5. It is obvious that the biosynthesized AgNPs displayed significant scavenging activity against DPPH, which increased in a dose-dependent

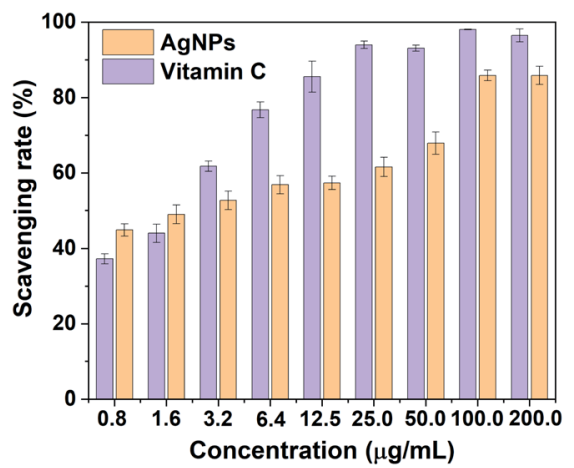

Fig. 5 Free radical scavenging rate of biosynthesized AgNPs and vitamin $\mathrm{C}$ against DPPH. 
manner. The radical scavenging rates were $44.8 \%$ and $85.8 \%$ in 0.8 and $100.0 \mu \mathrm{g} \mathrm{mL}^{-1}$ of AgNPs, respectively. This is comparable to vitamin C (Fig. 5), indicating that the biosynthesized AgNPs by RBM extract could be as free radical scavengers and used in treatment of many diseases caused by oxidative stress.

\subsection{Antibacterial activities of biosynthesized AgNPs}

Both Gram positive bacteria $S$. aureus and Gram negative bacteria $E$. coli were selected for evaluating the antibacterial activity of biosynthesized AgNPs. As shown in Fig. 6, the absorbance at $600 \mathrm{~nm}$ for these two pathogens with different doses of AgNPs were plotted over 9.0 hours. The antibacterial activity of AgNPs against both pathogens exhibited potential dose-dependent manner. The growth inhibition was clearly observed even in $0.8 \mu \mathrm{g} \mathrm{mL} \mathrm{m}^{-1}$ of AgNPs. The minimum inhibition concentrations (MIC) of as-prepared AgNPs were both 50.0 $\mu \mathrm{g} \mathrm{mL} \mathrm{m}^{-1}$ for E. coli and $S$. aureus, where both pathogens could be effectively inhibited within 9.0 h. The MIC values of AgNPs for both the $E$. coli and $S$. aureus were much smaller than that synthesized by Sea buckthorn extract $\left(400 \mu \mathrm{g} \mathrm{mL}{ }^{-1}\right),{ }^{21}$ where the AgNPs were spherical with an average particle size of $27.3 \pm$ $0.2 \mathrm{~nm}$. Otherwise, the AgNPs synthesized by KÜP and his coworkers $^{\mathbf{1 1}}$ using the leaf extract of Aesculus hippocastanum had bigger average size $50.0 \pm 5.0 \mathrm{~nm}$, but exhibited stronger antibacterial effects for both pathogens with MIC of $1.56 \mu \mathrm{g}$ $\mathrm{mL}^{-1}$. It seems that the toxic effects of biosynthesized AgNPs for bacterial strain have an ambiguous relationship with the average size. We speculated that the differentiated results on antibacterial activity might arise from different capped chemical constituents on biosynthesized AgNPs, which may lead to different mechanisms of cell death (see below).
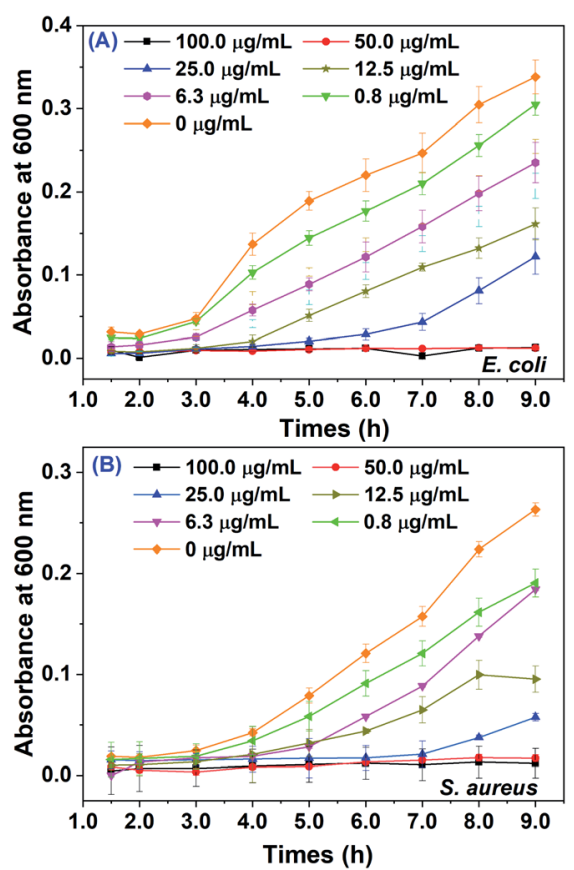

Fig. 6 The antibacterial activity of biosynthesized AgNPs for (A) E. coli and (B) S. aureus.

\subsection{Anticancer activities of biosynthesized AgNPs}

We preliminarily assessed the anticancer activity of biosynthesized AgNPs by using MTT assay. Three cancer cell lines including HCT116, HepG2 and HeLa were exposed to AgNPs with concentration in the range of 0 to $100.0 \mu \mathrm{g} \mathrm{mL}^{-1}$ for $24 \mathrm{~h}$. Fig. 7 displayed the cytotoxicity of biosynthesized AgNPs for these three cancer cell lines, where the cell viability for AgNPs treated cell lines occurred in a dose-dependent fashion. The cell viability after treating with AgNPs significantly decreased with increased doses of AgNPs. In the cases of HCT116, HepG2 and Hela cell lines, after incubating with 12.5, 25.0 and $20.0 \mu \mathrm{g}$ $\mathrm{mL}^{-1}$ of AgNPs, the cell viability would be significantly decreased to $41.1 \%, 16.4 \%$ and $48.1 \%$, respectively, meaning the superior anticancer activities. By using the GraphPad Prism 8.0 software package, the $\mathrm{IC}_{50}$ values of AgNPs against these three cancer cell lines were further determined to be 13.07, 19.67 and $26.18 \mu \mathrm{g} \mathrm{mL} \mathrm{m}^{-1}$, respectively. These values were comparable to the AgNPs synthesized using traditional medicine with anticancer effect, such as Iresine herbstii $\left(51 \mu \mathrm{g} \mathrm{mL} \mathrm{L}^{-1}\right.$ for Hela), ${ }^{40}$ Sesbania grandiflora $\left(20 \mu \mathrm{g} \mathrm{mL}{ }^{-1}\right.$ for MCF-7), ${ }^{24}$ Cornus officinalis (20.68 and $69.72 \mu \mathrm{g} \mathrm{mL}{ }^{-1}$ for HCT116 and HepG2) $)^{22}$ and Sea buckthorn (8.77, 4.61, 14.59, 16.05 and 27.98 $\mu \mathrm{g} \mathrm{mL}{ }^{-1}$ for HCT116, SW620, HepG2, MCF-7 and HeLa), ${ }^{21}$ indicating that the AgNPs synthesized using RBM extract could be a potential candidate for anticancer drugs.

\subsection{Internalization of biosynthesized AgNPs}

Until now, three well-defined mechanisms for the toxic effects of nanoparticles against cells have been proposed: (i) cell wall and membrane damage, (ii) intracellular penetration and damage, and (iii) oxidative stress. ${ }^{\mathbf{1 4 , 4 1}}$ It is obvious that to assess the internalization of AgNPs into cells would be helpful for understanding the cell death mechanism. Earlier studies ${ }^{\mathbf{3 4 , 4 2}}$ have shown that the light scattering signals could be detected and provide valuable information about cellular effects during the exposure to nanoparticles. By collecting the scattered light at perpendicular direction $\left(90^{\circ}\right)$ of incident laser $(488 \mathrm{~nm})$, which was defined as side scattering channel (SSC), the uptake potential of nanoparticles in cells could be evaluated using flow cytometry. As the enhanced intracellular density after taking up

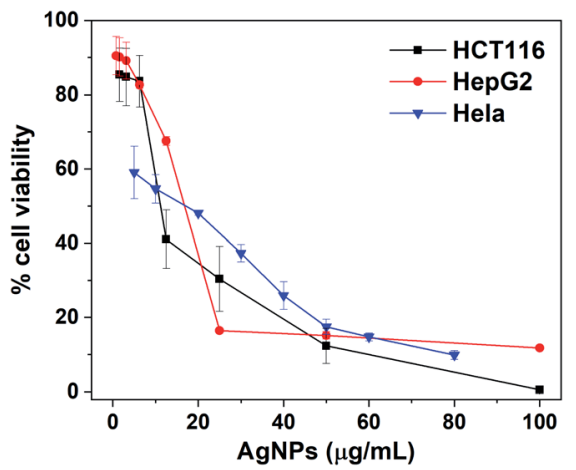

Fig. 7 Inhibition effects of biosynthesized AgNPs on HCT116 HepG2 and Hela cell lines. 
(A) E. coli
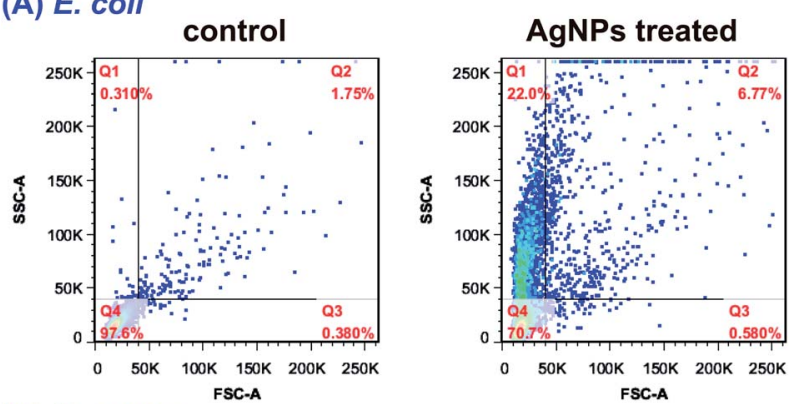

(B) S. aureus
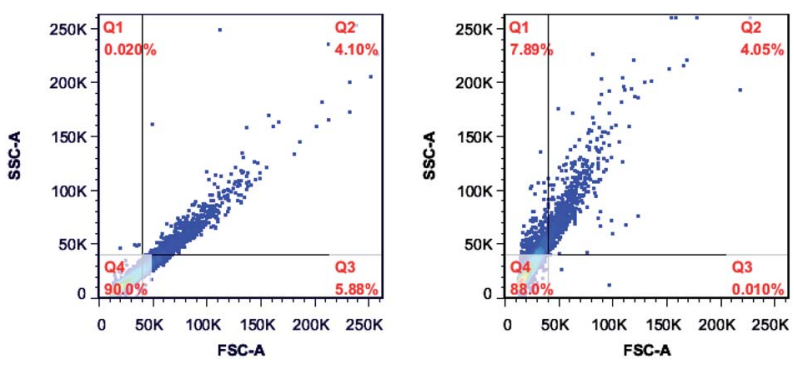

Fig. 8 Uptake analysis of AgNPs into (A) E. coli and (B) S. aureus assessed by flow cytometry.

particles into cells, SSC intensity will be consequently increased. Therefore, the flow cytometric analysis was performed to assess the internalization of AgNPs in bacteria. The density plot of flow cytometry light scatter before and after treatments of both E. coli and S. aureus with AgNPs $(5.0 \mu \mathrm{g}$ $\mathrm{mL}^{-1}$ ) for indicated times are shown in Fig. 8. Both pathogens treating with AgNPs displayed a marked increase in SSC intensity compared with control cells, indicating the internalization of synthesized AgNPs. Interestingly, the particles in E. coli were found to be showing a higher scattering in comparison to $S$. aureus suggesting the more uptake of biosynthesized AgNPs. The differentiated internalization of AgNPs maybe due to the distinct adherence to charged bacterial cell wall or the affinity for phosphorous and sulfur-containing compounds on the cell within a certain processing time. Considering the identical MIC of AgNPs against both pathogens, we speculated that the cell wall and membrane of $S$. aureus are susceptible to AgNPs biosynthesized by RBM.

The uptake analysis of AgNPs $\left(10 \mu \mathrm{g} \mathrm{mL}^{-1}\right)$ into HCT116, HepG2 and HeLa cancer cell lines were also performed. As shown in Fig. 9, the SSC values for these three cells do not have significant increase, indicating the negligible AgNPs' internalization. This result means that AgNPs biosynthesized by RBM cause necrosis possibly by disintegration of the cell wall and membrane, which is assumed to be primary mechanism of cell death. Briefly, nanoparticles adhesion to cell membrane would trigger cell morphological changes, and thus result in disruption of membrane permeability and respiratory functions via membrane depolarization. The increased membrane permeability and disruption of the cell wall would lead to the leak of cellular content including proteins, enzymes, DNA, ions, metabolites and energy reservoir into environment. The more precise mechanism of cell death needs more sophisticated experimental proof.

Herein, to successfully biosynthesize medical AgNPs by using Chinese herbal medicine residues (HMRs) have insightful implications for the development of both green synthesis of nanoparticles and the general recycling strategies for HMRs. The main points are as follows:

(1) There have some studies reported that the ingredients containing in HMRs would be changed after the decoction at high temperature. ${ }^{\mathbf{4 3 , 4 4}}$ This suggest that the HMRs is entirely
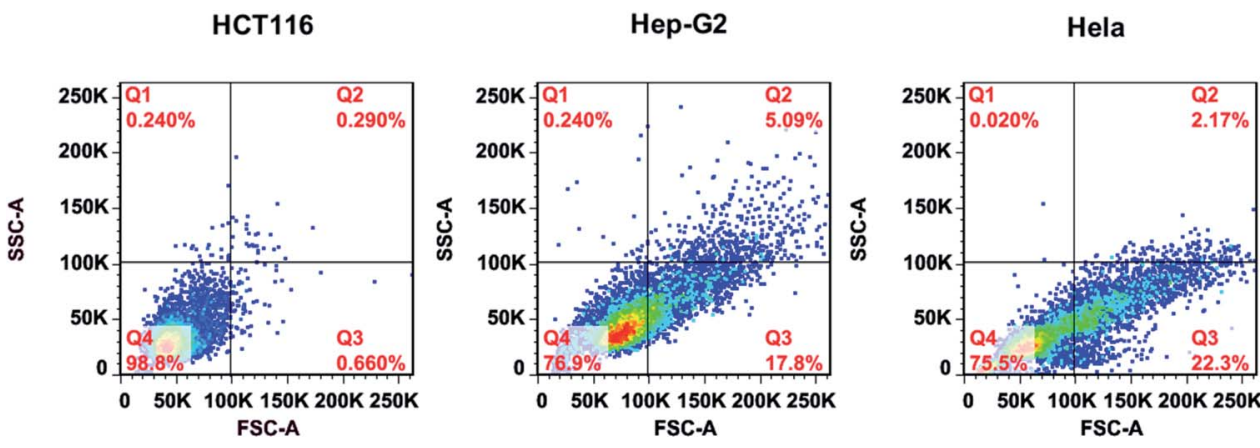

Control
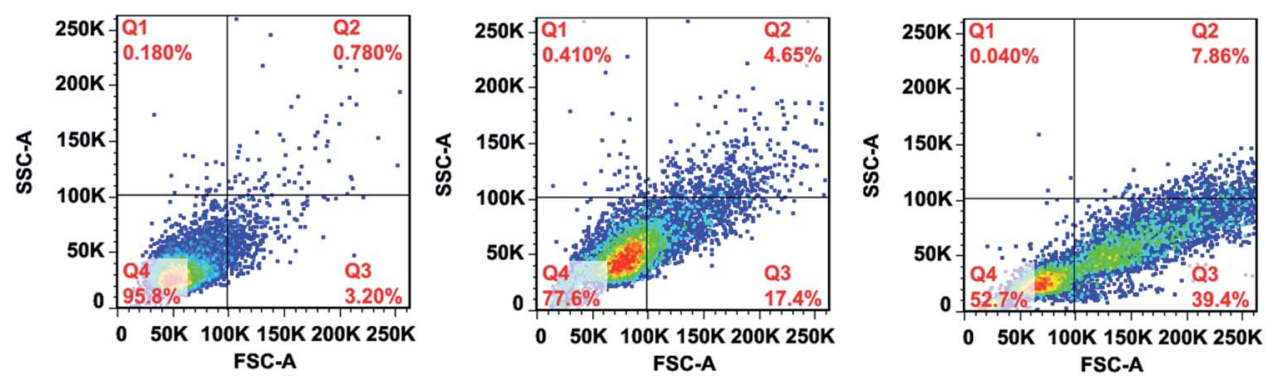

AgNPs treated

Fig. 9 Uptake analysis of AgNPs into HCT116, HepG2 and Hela cell lines assessed by flow cytometry. 
different from Chinese herbal medicine. To biosynthesize AgNPs using HMRs is span-new reaction system, but where the raw materials are more economical and easily achieved.

(2) It is also noteworthy that the HMRs is produced after decocting with water or ethanol, which make the medicinally active ingredients lose seriously. Generally, this is disadvantage for biosynthesizing AgNPs with superior medicinal activities. Obviously, to biosynthesize medicinal AgNPs using HMRs is a more challenging topic comparing with Chinese herbal medicine.

(3) We noticed that at present, the massive HMRs were directly disposed through stacking in pharmaceutical factories, abandoning randomly in the wild or landfilling and burning, which cause serious environmental pollution and the waste of resources. $^{27,45}$ Thus, a large number of efforts have been put into practice for general recycling strategies of HMRs. The existing dispose approach for HMRs are mainly co-compost, ${ }^{\mathbf{4}}$ feed additives, raw materials for papermaking, edible fungus cultivation, ${ }^{47}$ and ethanol preparation. ${ }^{30}$ These methods mainly considered the nutritional (organic matter, nitrogen, and phosphorus) benefits and texture like cellulose, lignin and hemicellulose, but ignored the abundant bioactive ingredients containing in HMWs. To synthesize AgNPs by utilizing bioactive ingredients containing in HMRs also provides novel strategy for HMRs reuse.

\section{Conclusions}

We present herein a novel approach for green synthesis of AgNPs by utilizing Bazheng Mixture residues under ultrasonic radiation at ambient temperature and systematically evaluate the biosynthesis parameters such as $\mathrm{pH}$, material proportion and incubation time on the formation of AgNPs. The dynamic light scattering (DLS) measurement revealed that the average size of biosynthesized AgNPs was susceptible to these parameters. However, the formation yield was almost impervious to the material proportion. The characterization of AgNPs synthesized under the most efficient parameters revealed that was a facecentered cubic (fcc) structure and spherical shape with an average particle size of $22.5 \pm 0.5 \mathrm{~nm}$ covered by anions (zeta potential: $-25.5 \mathrm{mV}$ ). The calculated free radical scavenging rate of AgNPs for DPPH was $85.8 \%$ in $100.0 \mu \mathrm{g} \mathrm{mL}^{-1}$ of AgNPs, indicating a good antioxidant activity. Furthermore, asprepared AgNPs also displayed superior toxic effect on both the bacteria and cancer cells. The IC $_{50}$ values for HCT116, HepG2 and HeLa cancer cell lines were 13.07, 19.67, and 26.18 $\mu \mathrm{g} \mathrm{mL}{ }^{-1}$, respectively. The MIC values of AgNPs for $E$. coli and $S$. aureus were both $50.0 \mu \mathrm{g} \mathrm{mL}{ }^{-1}$. By the uptake analysis of AgNPs into both bacteria and cancer cells, the mechanisms of cell death were preliminary disclosed. These results confirmed that the RBM could be a low-cost and eco-friendly resource for green synthesis of AgNPs, and provided a novel method for general recycling strategies of HMRs.

\section{Conflicts of interest}

There are no conflicts to declare.

\section{Acknowledgements}

This work was financially supported by the National Natural Science Foundation of China (Grant no. 21705029); the Young Talent fund of University Association for Science and Technology in Shaanxi, China (Grant no. 20190307) and the Fundamental Research Funds for the Central Universities, CHD (Grant no. 300102120303).

\section{Notes and references}

1 H. Kang, J. T. Buchman, R. S. Rodriguez, H. L. Ring, J. He, K. C. Bantz and C. L. Haynes, Chem. Rev., 2019, 119, 664-699.

2 S. Eckhardt, P. S. Brunetto, J. Gagnon, M. Priebe, B. Giese and K. M. Fromm, Chem. Rev., 2013, 113, 4708-4754.

3 Y. Chen, Z. Fan, Z. Zhang, W. Niu, C. Li, N. Yang, B. Chen and H. Zhang, Chem. Rev., 2018, 118, 6409-6455.

4 S. H. Lee and B. H. Jun, Int. J. Mol. Sci., 2019, 20, 24.

5 H. D. Beyene, A. A. Werkneh, H. K. Bezabh and T. G. Ambaye, Sustainable Mater. Technol., 2017, 13, 18-23.

6 L. Y. Wei, J. R. Lu, H. Z. Xu, A. Patel, Z. S. Chen and G. F. Chen, Drug Discovery Today, 2015, 20, 595-601.

7 X. X. Zhao, L. F. Zhou, M. S. R. Rajoka, L. Yan, C. M. Jiang, D. Y. Shao, J. Zhu, J. L. Shi, Q. S. Huang, H. Yang and M. L. Jin, Crit. Rev. Biotechnol., 2018, 38, 817-835.

8 N. Tarannum, Divya and Y. K. Gautam, RSC Adv., 2019, 9, 34926-34948.

9 H. E. Emam and H. B. Ahmed, Carbohydr. Polym., 2016, 135, 300-307.

10 V. Dhand, L. Soumya, S. Bharadwaj, S. Chakra, D. Bhatt and B. Sreedhar, Mater. Sci. Eng., C, 2016, 58, 36-43.

11 F. O. Kup, S. Coskuncay and F. Duman, Mater. Sci. Eng., C, 2020, 107, 11.

12 X. Huang, L. Chang, Y. Lu, Z. Li, Z. Kang, X. Zhang, M. Liu and D.-P. Yang, Mater. Sci. Eng., C, 2020, 113, 111015.

13 M. K. Rajesh, K. S. Muralikrishna, N. S. Swapna, K. B. Krishna, T. M. Subrahmanya, K. P. Sonu, K. Subaharan, H. Sweta, P. T. S. Keshava, N. Chandran, K. Indrani, K. B. Hebbar and A. Karun, Mater. Sci. Eng., C, 2020, 111, 110834.

14 A. Roy, O. Bulut, S. Some, A. K. Mandal and M. D. Yilmaz, RSC Adv., 2019, 9, 2673-2702.

15 E. Ituen, E. Ekemini, L. Yuanhua and A. Singh, J. Mol. Struct., 2020, 1207, 127819.

16 Y. Ping, J. Zhang, T. Xing, G. Chen, R. Tao and K.-H. Choo, J. Ind. Eng. Chem., 2018, 58, 74-79.

17 N. Skandalis, A. Dimopoulou, A. Georgopoulou, N. Gallios, D. Papadopoulos, D. Tsipas, I. Theologidis, N. Michailidis and M. Chatzinikolaidou, Nanomaterials, 2017, 7, 14.

18 G. Premanand, N. Shanmugam, N. Kannadasan, K. Sathishkumar and G. Viruthagiri, Appl. Nanosci., 2016, 6, 409-415.

19 T. V. M. Sreekanth, S. Ravikumar and I. Y. Eom, J. Photochem. Photobiol., B, 2014, 141, 100-105.

20 R. Sattari, G. R. Khayati and R. Hoshyar, Mater. Chem. Phys., 2020, 241, 122438. 
21 S. M. Wei, Y. H. Wang, Z. S. Tang, J. H. Hu, R. Su, J. J. Lin, T. Zhou, H. Guo, N. Wang and R. R. Xu, New J. Chem., 2020, 44, 9304-9312.

22 Y. Wang, S. Wei, K. Wang, Z. Wang, J. Duan, L. Cui, H. Zheng, Y. Wang and S. Wang, RSC Adv., 2020, 10, 27173-27182.

23 C. Dipankar and S. Murugan, J. Photochem. Photobiol., B, 2012, 98, 112-119.

24 M. Jeyaraj, G. Sathishkumar, G. Sivanandhan, D. MubarakAli, M. Rajesh, R. Arun, G. Kapildev, M. Manickavasagam, N. Thajuddin, K. Premkumar and A. Ganapathi, J. Photochem. Photobiol., B, 2013, 106, 86-92.

25 Y. Q. He, F. F. Wei, Z. Y. Ma, H. Zhang, Q. Yang, B. H. Yao, Z. R. Huang, J. Li, C. Zeng and Q. Zhang, RSC Adv., 2017, 7, 39842-39851.

26 D. Cyranoski, Nature, 2018, 561, 448-450.

27 Y. Zhou, A. Selvam and J. W. C. Wong, Bioresour. Technol., 2016, 217, 190-199.

28 S. J. Lee, S. Bose, J. G. Seo, W. S. Chung, C. Y. Lim and H. Kim, Clin. Nutr., 2014, 33, 973-981.

29 S. J. Ameh, O. O. Obodozie, U. S. Inyang, M. S. Abubakar and M. Garba, J. Med. Plants Res., 2010, 4, 72-81.

30 H. Z. Ma, H. G. Li, F. Y. Zhang, Q. H. Wang and M. B. Tu, Ind. Crops Prod., 2019, 128, 385-390.

31 Y. Zhou, A. Selvam and J. W. C. Wong, Bioresour. Technol., 2018, 249, 182-188.

32 M.-j. Zhang, K.-d. Chu and X.-l. Cheng, Chin. J. Integr. Med., 2009, 29, 46-50.

33 M. Sathishkumar, K. Sneha, S. W. Won, C. W. Cho, S. Kim and Y. S. Yun, J. Photochem. Photobiol., B, 2009, 73, 332-338.
34 H. Suzuki, T. Toyooka and Y. Ibuki, Environ. Sci. Technol., 2007, 41, 3018-3024.

35 H. Liu, H. Zhang, J. Wang and J. Wei, Arabian J. Chem., 2020, 13, 1011-1019.

36 G. Kaur, A. Kalia and H. S. Sodhi, Inorg. Nano-Met. Chem., 2020, 50, 35-41.

37 S. Khan, S. Singh, S. Gaikwad, N. Nawani, M. Junnarkar and S. V. Pawar, Environ. Sci. Pollut. Res., 2020, 27, 27221-27233.

38 R. Heydari and M. Rashidipour, Int. J. Breast Cancer, 2015, 2015, 846743.

39 N. Manosalva, G. Tortella, M. C. Diez, H. Schalchli, A. B. Seabra, N. Duran and O. Rubilar, World J. Microbiol. Biotechnol., 2019, 35, 9.

40 C. Dipankar and S. Murugan, J. Photochem. Photobiol., B, 2012, 98, 112-119.

41 L. Xu, Y. Y. Wang, J. Huang, C. Y. Chen, Z. X. Wang and H. Xie, Theranostics, 2020, 10, 8996-9031.

42 Y. Wu, M. R. K. Ali, K. Dansby and M. A. El-Sayed, Anal. Chem., 2019, 91, 14261-14267.

43 B. B. Wang, J. J. Ji, S. Zhao, J. J. Dong, P. Tan, S. S. Na and Y. G. Liu, Pharmacogn. Mag., 2016, 12, 4-8.

44 M. Wang, Y. Li, Y. Huang, Y. Tian, F. Xu and Z. Zhang, J. Sep. Sci., 2014, 37, 1148-1154.

45 X. Zeng, R. Y. Shao, F. Wang, P. W. Dong, J. Yu and G. W. Xu, Bioresour. Technol., 2016, 206, 93-98.

46 D. L. Wu, P. Liu, Y. Z. Luo, G. M. Tian and Q. Mahmood, J. Zhejiang Univ., Sci., B, 2010, 11, 497-505.

47 F. J. Meng, S. G. Yang, X. Wang, T. T. Chen, X. L. Wang, X. Y. Tang, R. J. Zhang and L. Shen, Journal of Infection and Public Health, 2017, 10, 749-754. 